DOI: https://doi.org/10.11144/Javeriana.umed61-3.spik

\title{
Estrategia de simulación para aplicar el protocolo SPIKES en la comunicación de malas noticias
}

\section{Simulation Strategy for the Application of SPIKES Protocol in the Communication of Bad News}

Recepción: 23/10/2019 | Aceptación: 27/01/2020

\author{
Gladys Cecilia Gordillo Navas \\ Médica cirujana, Universidad Pedagógica y Tecnológica \\ de Colombia. Especialista en Epidemiología, \\ Universidad de Boyacá. Profesora ad honorem de la \\ Pontificia Universidad Javeriana. Coordinadora de \\ Gestión Académica y líder de grupo de investigación \\ Salud San Rafael, ESE Hospital Universitario San \\ Rafael de Tunja, Colombia \\ Javier David Trujillo Martínez \\ Médico, Universidad de Ciencias Aplicadas y \\ Ambientales (UDCA). Miembro del semillero de \\ investigación Salud Familiar, UDCA. Integrante del \\ grupo de investigacion Salud San Rafael, ESE Hospital \\ Universitario San Rafael de Tunja, Colombia \\ Juan David Filizzola Bermúdez \\ Estudiante de Medicina, Pontificia Universidad \\ Javeriana de Bogotá. Miembro del semillero de Bioética \\ Clínica del Hospital Universitario San Ignacio. \\ Miembro del semillero Mente (Departamento de \\ Psiquiatría), Pontificia Universidad Javeriana, Bogotá,
}

Colombia

Correspondencia:

cedmedica@hospitalsanrafaeltunja.gov.co

Cómo citar: Gordillo Navas GC, Trujillo Martínez JD, Filizzola Bermúdez JD. Estrategia de simulación para aplicar el protocolo SPIKES en la comunicación de malas noticias. Univ. Med. 2020;61(3). https://doi.org /10.11144/Javeriana.umed61-3.spik

\section{RESUMEN}

En el campo de la salud, las malas noticias se definen como cualquier información que altere drástica y negativamente la visión del paciente sobre su futuro. La comunicación de malas noticias es de gran relevancia en la práctica clínica; sin embargo, a pesar de su importancia, la formación de los profesionales de salud frente a la adquisición de esta habilidad es deficiente. La entrega hábil de malas noticias puede proporcionar comodidad para el paciente y su familia; no obstante, una mala comunicación puede generar reacciones emocionales negativas, perspectivas erróneas sobre el pronóstico clínico e incluso tener implicaciones legales. La literatura describe varios protocolos y herramientas que favorecen la adecuada entrega de malas noticias, así como estrategias académicas que facilitan su aprendizaje. Este artículo propone una reflexión sobre el uso de escenarios simulados para el entrenamiento de profesionales de la salud, en comunicación de malas noticias, utilizando el protocolo SPIKES.

Palabras clave

simulación; comunicación; educación en salud; paciente simulado; malas noticias; SPIKES. 


\begin{abstract}
Bad news in the health care environment are defined as pieces of information delivered to patients, that can drastically (and often negatively) modify their life course and expectations for the future. Even though communicating bad news has great relevance in the clinical scenario, the training health care professionals need to acquire this skill is often deficient or overlooked. Skilfull delivery of bad news can provide confort for patients and their families, while poor communication of such information can generate negative emotional reactions, wrong perspectives about clinical prognosis and even lead to legal troubles for practitioners. Many protocols and tools for successfully delivering bad news have been described in literature, as well as diverse academic strategies for teaching such tools. The present article proposes a reflection about the use of simulated scenarios for training of health care professionals in bad news delivery, using the SPIKES protocol.
\end{abstract}

Keywords

simulation; communication; heatlh education; simulated patient; bad news; SPIKES protocol.

\section{Introducción}

Existen numerosas definiciones de malas noticias en el campo de la salud (1). Clásicamente, corresponden a cualquier información que afecte de modo negativo la percepción o la expectativa de un individuo sobre su curso de vida $(2,3)$. A su vez, dicha información alterará la visión de un paciente y su núcleo familiar, lo que dará como resultado respuestas cognitivas, conductuales y emocionales particulares (4). Esta es una tarea compleja y estresante (5), ya que en todos los casos implica un cambio en el curso de la trayectoria vital y el estilo de vida del paciente (1).

Aunque las malas noticias aparecen sobre todo en el contexto del diagnóstico de cáncer $\mathrm{u}$ otras enfermedades terminales, también pueden incluir información relacionada con el diagnóstico de una enfermedad crónica, una enfermedad que altera radicalmente la vida o una lesión irreversible que conduce a un cambio significativo en el estilo de vida del paciente (4).

El que una mala noticia se defina como tal no depende del contenido de la información o de su magnitud, sino de su repercusión en los pacientes y sus familias (6); por tanto, el profesional de salud no podrá estimar su efecto
(1), ya que dependerá del contexto de la vida de cada persona y de su capacidad para ejercer procesos de resiliencia (6).

Comunicar de manera adeacuada una mala noticia a una persona es parte esencial de la práctica profesional $(1)$. Hay evidencia $(7,8)$ de que una buena comunicación con esta y su familia puede ayudar a mejorar la calidad de vida, porque ello influye en una mejor percepción del paciente de su cuadro patológico, adherencia terapéutica y demás aspectos de su cuidado.

Sin embargo, dar una mala noticia es una tarea compleja dentro de la práctica profesional, y el duelo experimentado por los pacientes configura una respuesta natural, el cual no es un proceso rápido ni sencillo de enfrentar (6). A pesar de la importancia de esta habilidad en la práctica clínica, es limitada la educación formal en las escuelas del área de la salud (2). Se han generado manuales, protocolos y cursos (9); todos comparten algunas similitudes. Entre estos se encuentra el modelo SPIKES, como es el más empleado en los escenarios clínicos (2).

El protocolo SPIKES de seis pasos hace hincapié en seis componentes en el proceso de entrega de malas noticias (10): su fundamento es el concepto de que las malas noticias se reciben mejor cuando se entregan como un evento planificado, pues mejora cómo se recibe la información (3).

Por otro lado, tradicionalmente, la formación médica ha dependido del contacto con el paciente (11). En ese orden de ideas, para mejorar el entrenamiento clínico, los educadores utilizan pacientes reales, pacientes simulados y juegos de roles entre estudiantes como estrategias didácticas (12). La simulación con recursos de dramatización es una de las estrategias de enseñanza y se ha convertido en una herramienta fundamental para la formación de profesionales del área de la salud. Esta práctica permite modelar eventos clínicos en un ambiente seguro y controlado, lo que genera beneficios para el aprendizaje y y le ofrece al estudiante la posibilidad de desarrollar competencias prácticas, razonamiento crítico, toma de decisiones, trabajo en equipo, y, sobre todo, contribuir al fortalecimiento de la 
autoeficacia (13). Los pacientes simulados son un recurso valioso para enseñar y evaluar las habilidades de comunicación (11), permiten una retroalimentación en vivo y a posteriori por parte del paciente simulado, lo que enriquece el proceso de enseñanza-aprendizaje (13).

El entrenamiento y las capacitaciones en dar malas noticias han evidenciado que se mejora el desempeño de esta tarea (8). El presente artículo reflexiona sobre el abordaje en la entrega de una mala noticia, en el contexto del paciente simulado, bajo el protocolo SPIKES.

\section{Aspectos teóricos/metodológicos/ conceptuales}

El modelo de toma de decisiones centrado en el paciente, descrito desde fines del siglo $\mathrm{XX}$, provocó la divulgación de varios consensos de expertos para ayudar a los profesionales de la salud a transmitir malas noticias (4). Desde entonces, se incrementó la publicación bibliográfica en torno a comunicar malas noticias (9). No obstante, también se ha registrado una mínima capacitación en habilidades de comunicación en malas noticias en las escuelas de ciencias de la salud (8); se ha documentado que los profesionales en formación no reciben un entrenamiento formal para poder brindar malas noticias de forma adecuada (6). De hecho, la muerte - relacionada como una noticia de impacto negativo, y a pesar de que es un estado natural - es un tema prohibido en la sociedad y los profesionales en salud no reciben entrenamiento para lidiar con el fin de la vida (14).

Esta habilidad es importante ya que, dado el contexto, casi todos los profesionales de la salud tendrán que dar malas noticias durante su ejercicio laboral (2). La comunicación subóptima tiene consecuencias clínicas (8). La incapacidad de entregar malas noticias de manera efectiva y veraz puede conducir a la confusión del paciente (4). La calidad de la información, así como su manera de emisión, puede generar erróneamente decisiones basadas en supuestos poco realistas de su enfermedad (8), al igual que desencadenar problemas de adaptación frente a la enfermedad, otros problemas físicos y psicológicos (como estrés o incluso a un aumento de la ansiedad y depresión) $(1,8)$ no solo por parte del paciente, sino también la aparición de síntomas afectivos para el profesional de la salud (1).

Dosanjh et al. (15) refieren que las principales barreras por parte de los médicos para dar malas noticias son: los juicios de valor negativos que pueden emerger del contexto, como es sentir ineficacia e impotencia para curar la enfermedad del paciente; al igual que el miedo a no estar preparados para manejar las reacciones del paciente, a ser percibidos por estos o sus familias como personas poco empáticas e incompetentes o a cometer errores o ser mal entendidos durante la atención.

La evidencia sugiere que la carga afectiva de los profesionales no disminuye con los años de práctica o experiencia en la entrega de malas noticias (4). En relación con esto, se ha descrito una marcada afectación emocional, incluso casos de síndrome de desgaste profesional (burnout), que pueden generar una alta morbilidad psicológica en los profesionales de la salud (9).

Los pacientes y sus familias desean información honesta y directa cuando se entregan malas noticias. Por tal motivo, cuando no existe un entendimiento con el equipo de atención médica, el resultado es la desconfianza, entre otros desenlaces negativos de la atención, que se correlacionan con mayores tasas de demandas por negligencia médica $(3,8)$.

Generalmente, la capacitación de los equipos de atención médica en la entrega de malas noticias depende de las experiencias de los mentores (16). Según los hallazgos del estudio de Oikonomidou et al. (17), todos los participantes basaban su experticia en comunicación de malas noticias en la experiencia personal, más que en procedimientos recomendados o estandarizados. Los médicos declararon que ni ellos, ni las organizaciones de atención médica para las que trabajaban, siguieron un proceso de comunicación constante sobre la entrega de malas noticias. De su introspección se evidenció gran falta de conocimiento y habilidades con respecto a las formas óptimas de dar malas 
noticias y la comunicación general con los pacientes. Dado lo anterior, la entrega de malas noticias exige estrategias específicas de entrenamiento, teniendo en cuenta que, además, la literatura describe que las habilidades en dicho terreno no mejoran con el simple paso del tiempo y la práctica clínica (14).

Alrededor del mundo, el panorama en el entrenamiento y el dominio de la comunicación en malas noticias presentan un considerable vacío de habilidades en este tema. Según el estudio de Abbas et al. (18), el $40 \%$ de los médicos que trabajan en entornos de cuidados paliativos en Pakistán no daba una mala noticia correctamente. Otro estudio realizado por Jameel et al. (19) evidenció que el $85 \%$ de los participantes no se sentía cómodo para dar las malas noticias, y para lo hallado por Sarwar et al. (2), el $65 \%$ de los participantes estaba ansioso por recibir capacitación en este sentido. Así concluyeron que la mayoría de los residentes tenían poca satisfacción con respecto a sus habilidades en comunicar malas noticias (2).

La tarea de la entrega de malas noticias puede ser mejorada a través de entender el proceso que involucra el enfoque de protocolos de procedimiento, aplicando principios bien establecidos de comunicación y consejería (1). Para el estudio de Karam et al. (5), el 56,2 \% calificó su competencia en comunicar malas noticias como buena y muy buena antes del taller; sin embargo, esta perspectiva aumentó a un 93,7\% después de la sesión educativa. Así mismo, para lo hallado por Eid et al. (8), donde se realizó una sesión educativa diseñada para mejorar las habilidades de comunicación, el puntaje promedio de la clase en la lista de verificación de desempeño mejoró del 56,6\% antes de la intervención al 68,8\% después de la intervención $(\mathrm{p}<0,005)$. Por tal razón, es claro que la educación de los profesionales de la salud en este ámbito mejora sus estrategias de comunicación en la entrega de malas noticias, haciendo imprescindible su entrenamiento en estas habilidades.

La literatura describe la simulación como una herramienta del proceso de enseñanzaaprendizaje, ampliamente utilizada en la formación y el perfeccionamiento de habilidades de los profesionales del área de la salud (13). Cuando se utilizan escenarios o simulaciones para la entrega de malas noticias, los miembros del equipo médico informan una mayor apropiación y responsabilidad frente al paciente (3), y cuando existe un ejercicio de autoevaluación, los estudiantes que son conscientes de sus habilidades, son más estratégicos y tienen un mejor rendimiento que aquellos que no son conscientes de emitir un juicio crítico en la entrega de una mala noticia (20).

Con el fin de favorecer el proceso formativo, en 1960 se introdujo el término paciente simulado (11), una persona normal que simula ser un paciente real (21). Su uso en la educación en salud es globalmente aceptado como un medio valioso y efectivo para enseñar habilidades de comunicación, (22), tanto para estudiantes como para profesionales de la salud (23). Su participación permite retroalimentación conductual (11), pues se fortalece el dominio sobre las habilidades y conocimientos clínicos esenciales antes de ingresar en un entorno clínico real $(11,21)$. Sin embargo, oculta un verdadero problema en la formación integral de los profesionales de salud y genera que cada día se desvalorice la práctica profesional por errores en la comunicación.

Los temas comunes de los protocolos diseñados para comunicar malas noticias incluyen establecer un entorno apropiado, determinar el conocimiento previo del paciente frente a su condición clínica y el deseo de obtener detalles, evitar tecnicismos y eufemismos, apoyar las emociones del paciente, permitir preguntas, determinar los próximos pasos e, incluso, hacer un seguimiento de su caso (4). Aunque existen diversos protocolos y modelos establecidos, el más usado y ampliamente descrito es el modelo SPIKES (2).

En el estudio de Ferreira da Silveira et al. (24) se evaluaron los aspectos del modelo SPIKES en 160 profesionales del área de la salud en un hospital de Belo Horizonte, a partir de los cuales se evidenció que los participantes tenían fortalezas como usar un lenguaje comprensible $(85,95 \%)$; no obstante, 
presentaron inconsistencias al dar detalles sobre el tema (33,88\%), empatía ante la situación $(28,93 \%)$ y resolución de dudas $(51,24$ $\%)$. Ninguno conocía algún instrumento o herramienta que pudiera ayudar a abordar las malas noticias y transmitirlas a los pacientes, y la mayoría de ellos había aprendido observando a otros especialistas (42,15\%). Al final, los profesionales analizados identificaron que agregar el tema a los cursos de pregrado era importante $(45,45 \%)$ o muy importante $(42,15$ $\%)$.

Por otro lado, Flores et al. (25) llevaron a cabo un taller bajo la modalidad de videos y debriefing (sesión de análisis y reflexión), sobre la importancia de la comunicación de malas noticias. De manera breve, explicaron el protocolo SPIKES a 135 personas, médicos profesionales y en formación. Se les realizó un cuestionario pretaller en el cual se destacó que 117 participantes $(92,9 \%)$ no utilizaba ninguna metodología específica y 99 (79,2 \%) no habían recibido nunca formación en comunicación de malas noticias y 113 participantes $(89,7$ \%) no conocía el protocolo SPIKES. Al realizar la encuesta de satisfacción tras la realización del taller, 112 participantes $(85,5 \%)$ contestaron que consideraban muy importante su entrenamiento. Todos los participantes encontraron útil el taller, y 95 de ellos $(72,5$ $\%)$ consideraron que era muy útil; por lo se concluyó cuán importante es el entrenamiento en comunicación de malas noticias bajo una metodología práctica e interactiva.

En una revisión sistemática hecha por Camargo et al. (26) se agruparon nueve estudios que trabajaron la enseñanza del protocolo SPIKES con "aprendizaje activo", que incluye técnicas en las que los estudiantes priorizan la construcción de conocimiento por sí mismos, como juegos de rol, discusiones y casos. Un punto compartido entre diversas estrategias fue la presencia de una instancia de retroalimentación, que los estudiantes valoraron positivamente.

A continuación, se realiza un ejercicio de reflexión sobre el abordaje en la entrega de una mala noticia, en el contexto del paciente simulado, bajo el protocolo SPIKES.
Aprender a dar malas noticias desde la mirada del paciente simulado

En general, los pacientes simulados son personas entrenadas para dramatizar situaciones clínicas específicas, como las que el estudiante o profesional de la salud deberá enfrentar en su práctica diaria (27). Para este fin se han empleado actores profesionales o en formación, estudiantes de medicina e incluso personas del común sin experiencia formal en actuación. Los pacientes simulados se han usado principalmente en el entrenamiento de habilidades de comunicación para el personal de la salud, situación en que los profesionales deben establecer una relación humana y empática con un paciente o familiar mientras entregan información clínica, muchas veces, de gran relevancia para la vida de las personas (27).

Para lograr que las simulaciones clínicas sean un ejercicio educativo efectivo que cumplan con su función de fortalecer en el aprendiz competencias esenciales, el paciente simulado debe cumplir con ciertos parámetros en su representación (27). En primer lugar, debe procurar ser preciso y realista, caracterizar a un paciente lo más parecido a uno real, teniendo en cuenta el contexto sociocultural al que se enfrentan los profesionales, la manera de expresarse, el entendimiento técnico de los procesos de salud-enfermedad que poseen las personas y el abanico de reacciones conductuales y emocionales de los pacientes ante la información recibida. En segundo lugar (y he aquí el motivo de utilizarse en ocasiones el término "paciente estandarizado") (27), la representación del paciente simulado debe ser consistente y repetible a lo largo del tiempo, de tal manera que distintos estudiantes expuestos al mismo escenario clínico simulado puedan cumplir de manera sistemática con los objetivos de aprendizaje propuestos.

En este sentido, el actor o paciente simulado debe empaparse de las situaciones clínicas a las que se expone a diario el personal de salud, intentar de alguna manera ponerse en los zapatos de un paciente o familiar a quien le 
es entregada información importante sobre su salud o sobre situaciones patológicas que podrían redirigir el curso de su vida. Así mismo, deberá ser receptivo y estar abierto a la información y contacto humano que ofrece quien participa en la simulación, pues las palabras, los gestos y las actitudes del participante condicionarán y guiarán las respuestas del actor. Cada simulación, al fin y al cabo, aunque deba cumplir con ciertos estándares y ser replicable entre participantes, es un encuentro único e irrepetible entre actorpaciente simulado y participante-aprendiz. Tal vez, parte del éxito del encuentro esté en que el actor se permita reaccionar de manera particular ante cada una de las personas que participan en la simulación.

En particular para el caso de la comunicación de malas noticias, se ha observado que el uso de protocolos estandarizados como el SPIKES genera mayor seguridad y confianza a la hora de que los profesionales de la salud entreguen este tipo de información. Una metodología ya empleada para el aprendizaje de dichos protocolos es el uso de escenarios simulados. Eid et al. (8), por ejemplo, utilizaron dicha estrategia e incluso involucraron a los pacientes simulados en la evaluación de los participantes, diseñando una "lista de chequeo para entregar malas noticias", basada en el protocolo SPIKES que permitiese la evaluación estandarizada de los participantes.

Siguiendo la idea de involucrar a los actorespacientes simulados como fichas clave en el proceso de aprendizaje, y en miras a darles a las simulaciones replicabilidad y fiabilidad, podría entonces enseñárseles no solo a los participantes, sino también a los pacientes simulados en qué consisten específicamente dichos protocolos. Al aplicarlos, se espera que el aprendiz cumpla con ciertos pasos para la entrega de malas noticias una vez entra en contacto con el actor; no obstante, si el paciente simulado es consciente de dichos pasos y ha estudiado el protocolo, de manera tácita y manteniendo la verosimilitud de la representación, guiaría al participante dentro del cumplimiento del protocolo, a través de herramientas propias de su entrenamiento, como el uso de guiones semiestructurados y la disposición para la improvisación en cada encuentro.

Se propone, entonces, que el actor conozca los pasos que (según el protocolo SPIKES) debiera seguir cada participante en el momento de comunicar una mala noticia, y teniendo una especie de guión semiestructurado, reaccione de determinada manera según quién participa en la simulación vaya o no cumpliendo los pasos.

Así, puesto en el papel de quien recibe una mala noticia (por ejemplo, la muerte de un familiar), el actor permitiría una conversación más fluida y atenta con el participante siempre que este vaya cumpliendo los pasos propuestos por el SPIKES; mientras que lo interrumpiría, se molestaría o exigiría modificaciones en la comunicación si observa que el participante pasa por alto o se aleja demasiado de alguno de los elementos del protocolo.

Surge, sin embargo, la cuestión de si el hecho de ceñirse tan estrictamente a uno de muchos protocolos disponibles para comunicar malas noticias, además de estandarizar respuestas "positivas y negativas" ante la conducta del participante, iría en detrimento de la verosimilitud de la representación del escenario clínico. Es claro, por una parte, que aun cuando existan metodologías estandarizadas como el protocolo SPIKES, con el tiempo cada persona desarrollará su manera particular de comunicar malas noticias y el hecho de que no se ciña a un protocolo determinado no le restará validez o efectividad. Por otra, el actor se enfrentaría al reto de guiar muy sutilmente al participante en el cumplimiento de los pasos para la comunicación de malas noticias, sin que este último se diera cuenta de que está siendo guiado.

Llevando la reflexión a un absurdo, es claro que en la práctica real ningún paciente o familiar le recordaría al médico que está omitiendo uno de los pasos del protocolo SPIKES para la entrega de malas noticias. En el escenario simulado, el actor deberá encontrar maneras muy sutiles (verbales o no verbales) de hacer caer en cuenta al participante que debe ajustar algún elemento en su comunicación o que omitió algún paso clave definido por el protocolo. Ello le permitirá, así mismo, modificar su comunicación o reorientar 
su discurso durante la misma simulación. El uso entonces de un guión semiestructurado - como el que se propone a continuación- no implica caer en una representación repetitiva y poco realista del escenario clínico; más bien, se trata de una herramienta (que por definición debería ser flexible) que le permita al actor relacionarse con el modelo de comunicación de malas noticias, reaccionar según se cumplan o no los objetivos establecidos y generar así correspondencia con el participante en un escenario similar al real, donde al final se puede retroalimentar sobre lo ocurrido (tabla 1).

\section{Tabla 1.}

Ejemplo de guion semiestructurado para paciente simulado, bajo el protocolo SPIKES

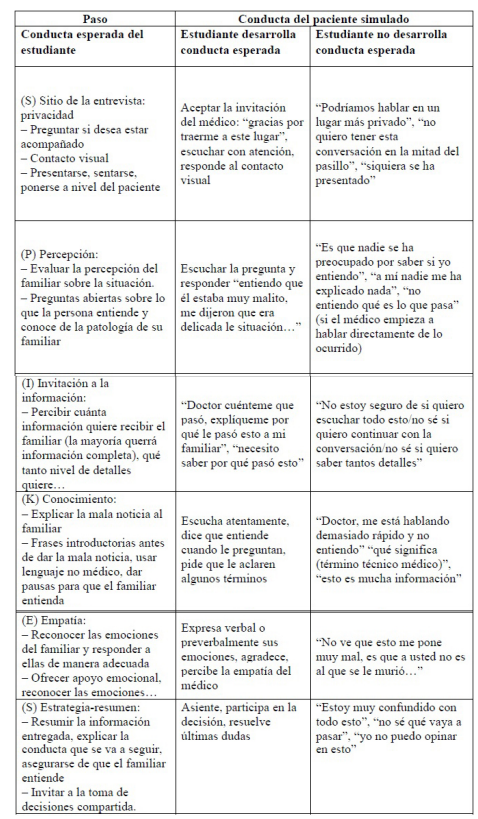

Nota: Se propone un ejemplo en el que el paciente simulado es una persona a quien se le debe entregar la mala noticia de que su familiar falleció o se encuentra gravemente enfermo.

Según el estudiante cumpla con los pasos propuestos por el protocolo SPIKES, el actor reaccionará de manera positiva y permitirá que se siga desarrollando el encuentro (segunda columna). Por el contrario, si el participante omite o va en contra de algunos de los pasos, el actor reaccionará a modo que se corrijan las falencias antes de continuar (tercera columna).

\section{Conclusiones}

La principal función del personal de salud es acompañar al paciente y a su familia hasta el final de su ciclo vital, encaminando sus acciones en actividades de bienestar y advirtiendo los futuros riesgos por conductas no saludables. Por tal razón, no es justificable que solo se enmarquen las acciones profesionales en salud hasta un punto de formulación.

En algún momento de su vida, cada profesional se tendrá que ver abocado a brindar una mala noticia, y si bien esto ha sido un proceso de erroraprendizaje, por estar inmersos en la atención hospitalaria o por el desconocimiento del alcance de un "valde de agua fría", el paciente como tal no solo vive su enfermedad, sino el proceso de enfrentarse a ella y asumir las repercusiones que tiene en su vida $(1,8)$.

La estrategia de la enseñanza del protocolo SPIKES bajo la mirada del paciente simulado es fácilmente replicable y útil. Su uso favorece el aprendizaje de la comunicación de malas noticias en un ambiente seguro, donde el participante se enfrenta a un escenario similar al real y tiene la posibilidad de recibir una retroalimentación del ejercicio, para que reflexione así sobre su conducta y optimice de tal forma el entrenamiento en este tipo de habilidades.

Grandes cambios esperan a las nuevas generaciones de profesionales de salud; pero están en ellas, en los procesos educativos en salud, corregir su marcha y enfocar la atención no solo en tratar una patología, sino en el valorar a un ser humano con sus circunstancias particulares que van hasta sus últimos días (19).

Se requiere promulgar y proponer, por tanto, constructos académicos dinámicos y multidimensionales, con estrategias que vayan más allá de la enseñanza de conocimientos en un aula de clases, que se enfoquen en el entendimiento del dolor humano y su abordaje, no solo desde la visión de la no maleficencia, sino del alcance real de estar en un momento de hecatombe al entregar una mala noticia. 


\section{Agradecimientos}

Agradecemos a Dios todo poderoso, que nos permite continuar día a día con nuestros propósitos y metas. A nuestras familias, por apoyarnos en la hermosa labor de la medicina.

\section{Referencias}

1. Herrera A, Ríos M, Manríquez JM, Rojas G. Entrega de malas noticias en la práctica clínica. Rev Med Chil [Internet]. 2014;142 (10):1306-15. Disponible en: http s://scielo.conicyt.cl/scielo.php?script $=$ sci arttext\&pid $=$ S0034-98872014001000011

2. Sarwar MZ, Rehman F, Fatima SM, et al. Breaking bad news skill of postgraduate residents of tertiary care hospital of Lahore, Pakistan: A crosssectional survey. J Pak Med Assoc [Internet]. 2019;69(5):695-9. Disponible en: https://www.semanticscholar.org/paper /Breaking-bad-news-skill-of-postgraduate-r esidents-A-Sarwar-Rehman/cc5609b398e8 fa55a51f39fe26d378a8bb55cd14

3. Nielsen PE, Munroe M, Foglia L, et al. collaborative practice model: improving the delivery of bad news. Clin J Oncol Nurs. 2012;22(1):23-7.

4. Franklin J, Berkey D, Joseph P, et al. Delivering bad or life-altering news. Am Fam Physician [Internet]. 2018;98(2):99-104. Disponible en: http://www.embase.com/search/results? subaction $=$ viewrecord $\&$ from $=$ export\&id $=\mathrm{L} 623051532$

5. Karam VY, Barakat H, Aouad M, et al. Effect of a simulation-based workshop on breaking bad news for anesthesiology residents: An intervention study. BMC Anesthesiol. 2017;17(1):4-9.

6. Huarcaya-Victoria J, Infanto SD, Nacional H, et al. El duelo y cómo dar malas noticias en medicina. An Fac med [Internet]. 2019;80(1):86-91. Disponible en: http://w ww.scielo.org.pe/scielo.php?script $=$ sci_artt ext\&pid $=$ S1025-55832019000100016
7. Martín Hernández I, Trujillo Matienzo C. Comunicación de malas noticias a pacientes con enfermedades neurodegenerativas: habilidades del personal médico. Rev Latinoam Bioética [Internet]. 2009;9(2):77-85. Disponible en: http://portal.amelica.org/ameli/jatsRep o/127020306006

8. Eid A, Petty M, Hutchins L, et al. Breaking bad news: Standardized patient intervention improves communication skills for hematology-oncology fellows and advanced practice nurses. J Cancer Educ. 2009;24(2):154-9.

9. Ramírez-Ibáñez MT, Ramírez-de la Roche OF. Cómo comunicar malas noticias en la práctica médica. Atención Fam [Internet]. 2015;22 (4):95-6. Disponible en: https://ww w.elsevier.es/es-revista-atencion-familiar-2 23-articulo-como-comunicar-malas-notici as-practica-S1405887116300608

10. Baile W, Buckman R, Lenzi R, et al. SPIKES-a six-step protocol for delivering bad news: Application to the patient with cancer. Oncologist [Internet]. 2000;5(4):302-11. Disponible en: http://theoncologist.alpham edpress.org/content/5/4/302.long

11. Cleland JA, Abe K, Rethans JJ. The use of simulated patients in medical education: AMEE Guide No 42 1. Med Teach. 2009;31(6):477-86.

12. Parvan K, Hosseini FA, Bagherian S. Simulated patient and role play methodologies for communication skills training in an undergraduate medical program: Randomized, crossover trial. Educ Heal [Internet]. 2018;31:10-6. Disponible en: http://www.educationforhealth.net/arti cle.asp?issn $=1357-6283$; year $=2018$; volu me $=31$; issue $=1 ;$ spage $=10$; epage $=16$; aula st $=$ Taylor

13. Negri EC, Mazzo A, Martins JCA, et al. Simulação clínica com dramatização: Ganhos percebidos por estudantes e profissionais de saúde. Rev Lat Am Enfermagem. 2017;25. http://dx.doi.org/10 $.1590 / 1518-8345.1807 .2916$ 
14. Payán G C. Comunicar malas noticias: una tarea difícil pero necesaria. Ciruped [Internet]. 2012;2(1):35-9. Disponible en: http://www.ciruped.org/articulos/vol2n 12012/arvol2n12012-4.pdf

15. Dosanjh S, Barnes J, Bhandari M. Barriers to breaking bad news among medical and surgical residents. Med Educ. 2001;35(3):197-205.

16. Brown VA, Parker PA, Furber L, et al. Patient preferences for the delivery of bad news: The experience of a UK Cancer Centre. Eur J Cancer Care (Engl). 2011;20(1):56-61.

17. Oikonomidou D, Anagnostopoulos F, Dimitrakaki C, et al. Doctors' perceptions and practices of breaking bad news: a qualitative study from greece. Health Commun. 2017;32(6):657-66.

18. Abbas S, Muhammad S, Mubeen S, et al. Awareness of palliative medicine among Pakistani doctors: a survey. J Pak Med Assoc. 2004; 54:195-8.

19. Jameel A, Noor SM, Ayub S. Survey on perceptions and skills amongst postgraduate residents regarding breaking bad news at teaching hospitals in Peshawar, Pakistan. J Pak Med Assoc. 2012; 62:585.

20. Rat A, Ricci L, Guillemin F, et al. Development of an online formative selfassessment tool for physicians to practice breaking bad news: The breaking bad news Tool (BRADNET). JMIR Med Educ. 2018;4(2):1-27.

21. Beigzadeh A, Bahmanbijari B, Sharifpoor E, et al. Standardized patients versus simulated patients in medical education: are they the same or different. J Emerg Pract Trauma [Internet]. 2015;2(1):25-8. Disponible en: http://www.jept.ir/article_10855_cb64e 5d76610f95fd11365cde44e499b.pdf

22. Kaplonyi J, Bowles KA, Nestel D, et al. Understanding the impact of simulated patients on health care learners' communication skills: a systematic review. Med Educ. 2017;51(12):1209-19.

23. Laughey W, Sangvik Grandal N, M Finn G. Medical communication: the views of simulated patients. Med Educ. 2018;52(6):664-76.

24. Ferreira da Silveira FJ, Botelho CC, Valadão CC. Breaking bad news: doctors' skills in communicating with patients. Sao Paulo Med J. 2017;135(4):323-31. https://doi.org /10.1590/1516-3180.20160221270117

25. Flores-Funes D, Aguilar-Jiménez J, Lirón-Ruiz $\mathrm{R}$, et al. ¿Comunicamos correctamente las malas noticias en medicina?: resultados de un taller de formación basado en videos y debriefing. Educ Med. 2018. https://doi.org /10.1016/j.edumed.2018.07.005

26. Camargo N, Lima M, Brietzke E, Mucci S, Góis Aécio F. La enseñanza de cómo dar malas noticias: una revisión sistemática. Rev Bioét [Internet]. 2019 June [cited 2020 Jan 26];27(2):326-40. Disponible en: http:/ /www.scielo.br/scielo.php?script $=$ sci artte $\mathrm{xt}$ \&pid=S1983-80422019000200326̄\&lng =en. Epub July 01, 2019. http:// dx.doi.org/10.1590/1983-80422019272317

27. Wallace J, Rao R, Haslam R. Pacientes simulados y exámenes clínicos estructurados objetivos: revisión de su uso en educación médica: avances en el tratamiento psiquiátrico. Prensa de la Universidad de Cambridge. 2002;8(5):342-8. 